\title{
Characterization of Mycobacterium avium clinical isolates in Japan using subspecies-specific insertion sequences, and identification of a new insertion sequence, ISMav6
}

\author{
Correspondence \\ Kenji Ogawa \\ ogawak@toumei.hosp.go.jp
}

Received 9 December 2008

Accepted 20 March 2009

\author{
Kazuya Ichikawa, ${ }^{1,2}$ Tetsuya Yagi, ${ }^{3}$ Makoto Moriyama, ${ }^{1,2,4}$ \\ Takayuki Inagaki, ${ }^{1,2}$ Taku Nakagawa, ${ }^{5}$ Kei-Ichi Uchiya, ${ }^{1}$ Toshiaki Nikai ${ }^{1}$ \\ and Kenji Ogawa ${ }^{2,5}$
}
${ }^{1}$ Department of Microbiology, Faculty of Pharmacy, Meijo University, 150 Yagotoyama, Tempaku- ku, Nagoya, Aichi 468-8503, Japan
${ }^{2}$ Department of Clinical Research, National Hospital Organization, Higashinagoya National Hospital, 5-101 Umemorizaka, Meito-ku, Nagoya, Aichi 468-8620, Japan
${ }^{3}$ Department of Infectious Diseases, Center of National University Hospital for Infection Control, Nagoya University Hospital, 65 Tsurumai, Showa-ku, Nagoya, Aichi 466-8560, Japan
${ }^{4}$ Department of Pharmacy, National Hospital Organization, Nagoya Medical Center, 4-1-1 Sannomaru, Naka-ku, Nagoya, Aichi 460-0001, Japan
${ }^{5}$ Department of Pulmonary Medicine, National Hospital Organization, Higashinagoya National Hospital, 5-101 Umemorizaka, Meito-ku, Nagoya, Aichi 468-8620, Japan

Clinical isolates of Mycobacterium avium $(n=81)$ from patients with pulmonary infections who were HIV-negative and isolates $(n=33)$ from HIV-positive patients were subjected to genetic analysis by PCR detection of three $M$. avium-specific insertion sequences (IS901, IS1245 and IS1311), and nucleotide sequencing of the heat-shock protein 65 (hsp65) gene. All clinical isolates were identified as 'M. avium subspecies hominissuis' by sequence analysis of $h s p 65$. Compared with clinical isolates of $M$. avium reported elsewhere, IS1245 was found less frequently in Japanese isolates (96/114 isolates, $84 \%$ ) and IS901 was detected more frequently (76/114 isolates, $67 \%$ ). One isolate was found to lack IS1311, which has not been reported previously for ' $M$. avium subsp. hominissuis'. Nucleotide sequence analysis of the PCR products for IS901 revealed that all clinical isolates had the same new insertion sequence, designated ISMav6, which had 60 point mutations compared with the nucleotide sequence of the original IS901. These results suggest that ' $M$. avium subsp. hominissuis' with ISMav6 is prevalent in Japan. ISMav6 may have implications for the virulence of $M$. avium and contribute to an increase of $M$. avium infections in this country.

\section{INTRODUCTION}

Infection with the Mycobacterium avium complex has increased in recent years (Marras \& Daley, 2002). In Japan, the prevalence of $M$. avium complex disease has increased from 0.82 per 100000 persons in the 1970 s to 3.52 per 100000 , as shown by a recent epidemiological study

The GenBank/EMBL/DDBJ accession numbers for the ISMav6, heatshock protein 65 sequevar code 16 and heat-shock protein 65 sequevar code 17 sequences are AB447556, AB453830 and AB453831, respectively.

A figure showing the sequence alignment of ISMav6 and IS901 is available with the online version of this paper.
(Sakatani, 1999), which is considerably higher than the reported rate of 0.2 per 100000 in Europe (Henry et al., 2004). Pulmonary M. avium infection has been reported frequently among HIV-negative middle-aged women without any organic lung diseases. The human or bacterial factors contributing to the pathophysiology of $M$. avium infection and unresponsiveness to therapy remain to be elucidated.

M. avium comprises four subspecies that infect specific hosts and are differentiated by the presence or absence of specific insertion sequences. M. avium subsp. avium and M. avium subsp. silvaticum, isolated from birds, possess IS901 and low copy numbers of IS1245 (Dvorska et al., 2003; Kunze et al., 1992; Ritacco et al., 1998). 'M. avium subsp. hominissuis', 
from humans and swine, usually harbours high copy numbers of IS1245 and IS1311, but none of IS901 (Mijs et al., 2002). M. avium subsp. paratuberculosis, from ruminant livestock and wildlife, has IS900 but not IS1245 or IS901 (Chacon et al., 2004; Harris \& Barletta, 2001). However, it is difficult to determine definitively the type of subspecies by using insertion sequences, because some strains lack some specific insertion sequences (Turenne et al., 2007). Accordingly, classification based on the nucleotide sequence of hsp65 has been proposed (Turenne et al., 2006).

The purpose of this study was to characterize $M$. avium pathogenic clinical isolates recovered from patients with or without HIV infection in Japan using different molecular methods.

\section{METHODS}

Strains. M. avium subsp. avium (GTC00603, derived from M. avium ATCC 25291 and ATCC 35718), M. avium subsp. silvaticum (ATCC 49884) and ' $M$. avium subsp. hominissuis' (104 and ATCC 19978) were used as standard strains. The clinical isolates were 81 strains recovered from the sputum of $81 \mathrm{HIV}$-negative patients with pulmonary diseases, and 33 strains from HIV-positive patients, including 5, 27 and 1 recovered from sputum, blood and lymph node, respectively.

Identification of strains. The standard strains GTC00603 and ATCC 49884 were identified by detection of the three-band bird-type IS 1245 by RFLP analysis, and sequevar code 4 was confirmed by sequence analysis of the $3^{\prime}$ fragment of $h s p 65$.

Clinical isolates were identified as M. avium by using the COBAS AMPLICOR Mycobacterium Test (Roche Diagnostic Systems). In addition, sequence analysis of the $3^{\prime}$ fragment of the $h s p 65$ gene was performed to identify the subspecies of $M$. avium. The hsp65 sequevar codes of the subspecies were code 4 for $M$. avium subsp. avium, codes 1 , 2, 3, 7-9 and 15 for ' $M$. avium subsp. hominissuis', and codes 5 and 6 for M. avium subsp. paratuberculosis (Alvarez et al., 2008; Turenne et al., 2006).
PCR analysis. The identified strains were cultured in $5 \mathrm{ml} 7 \mathrm{H} 9$ liquid medium supplemented with $10 \%$ oleic acid-albumin-dextrosecatalase enrichment at $37{ }^{\circ} \mathrm{C}$ for $1-2$ weeks, and then transferred to $25 \mathrm{ml}$ of the same medium for further culture. The culture was centrifuged and then DNA was extracted with Isoplant II according to the manufacturer's recommendations (Nippon Gene). The extracted DNA was dissolved in $50 \mu \mathrm{l}$ Tris EDTA buffer (Sigma) and used as a template. Mixtures were prepared containing template DNA (5$50 \mathrm{ng})$, dNTP mixture $(5 \mu \mathrm{l}), 10 \times$ PCR buffer $(5 \mu \mathrm{l})$, each primer set (both $12.5 \mu \mathrm{M}$ ), $2.5 \mathrm{U}$ AmpliTaqGold (Applied Biosystems) and DMSO (Wako) to a final concentration of $4 \%$. Sterilized purified water was added to bring the total volumes up to $50 \mu \mathrm{l}$ and the mixtures were subjected to PCR. The PCR primers for IS901-1, IS1245, IS1311, IS901-flanking region 300 (FR300) and the hsp65 gene are shown in Table 1. The PCR program used consisted of an initial denaturing step at $95{ }^{\circ} \mathrm{C}$ for $10 \mathrm{~min}$, followed by 35 cycles of denaturing at $94{ }^{\circ} \mathrm{C}$ for $60 \mathrm{~s}$, annealing at $58{ }^{\circ} \mathrm{C}$ for IS901-1 and $h$ sp 65 or $68{ }^{\circ} \mathrm{C}$ for IS 1245 , IS 1311 and FR300 for $60 \mathrm{~s}$, extension at $72{ }^{\circ} \mathrm{C}$ for $60 \mathrm{~s}$, and then a final extension step at $72{ }^{\circ} \mathrm{C}$ for $7 \mathrm{~min}$.

The amplified DNA products were subjected to electrophoresis on a $2 \%$ agarose gel (Invitrogen) to determine their size (Table 1). Judgment was done by comparison with the size of the products of the standard strains as positive controls.

Sequence analysis. The PCR products of IS 901 and the $h s p 65$ gene were subjected to sequence analysis by the method of Turenne et al. (2006) with the same forward and reverse primers as used for PCR The nucleotide sequences of the $h s p 65$ gene thus obtained were compared with the reported data by BLAST analysis using the NCBI server (http://www.ncbi.nlm.nih.gov/). Alignment was performed with CLC Sequence Viewer 4.6.2 (CLC Bio). In order to determine the nucleotide sequence upstream and downstream of the PCR products of IS901, two primer sets (Table 1) were designed for IS9012 and IS901-3 using Primer3 (http://frodo.wi.mit.edu/). DNA was amplified under the same PCR conditions as above, and sequence analysis of each product was performed.

RFLP analysis. Probes were prepared by using a PCR DIG Probe Synthesis kit with the corresponding primers shown in Table 1, according to the manufacturer's recommendations (Roche). Four nanograms of template DNA extracted from each strain was digested

Table 1. Primers used for identification and characterization of the $M$. avium isolates

\begin{tabular}{|c|c|c|c|}
\hline Target gene & Product size (bp) & Nucleotide sequence & Reference \\
\hline IS901-1 & 1108 & $\begin{array}{l}\text { F: 5'-GCAACGGTTGTTGCTTGAAA-3' } \\
\text { R: 5'-TGATACGGCCGGAATCGCGT-3' }\end{array}$ & Kunze et al. (1992) \\
\hline IS901-2 (1-532) & 532 & $\begin{array}{l}\text { F: 5'-TTCCTTAGGGTTGAAGGGGT-3' } \\
\text { R: 5'-GTCGGTCTTGCCTTCACCT-3' }\end{array}$ & This work \\
\hline IS901-3 (970-1472) & 502 & $\begin{array}{l}\text { F: 5'-GGATCGCCAGATCAAGGATA-3' } \\
\text { R: 5'-TCTCAATGAGCGTGTCAAC-3' }\end{array}$ & This work \\
\hline FR300 & 300,1776 & $\begin{array}{l}\text { F: 5'-CAGCCAGCCGAATGTCATCC-3' } \\
\text { R: 5'-CAACTCGCGACACGTTCACC-3' }\end{array}$ & Nishimori et al. (1995) \\
\hline IS 1245 & 427 & $\begin{array}{l}\text { F: 5'-GCAACGGTTGTTGCTTGAAA-3' } \\
\text { R: 5'-AGGTGGCGTCGAGGAAGA-3' }\end{array}$ & Johansen et al. (2005) \\
\hline IS 1311 & 198 & $\begin{array}{l}\text { F: 5'-GCGTGAGGCTCTGTGGTGAA-3' } \\
\text { R: 5'-ATGACGACCGCTTGGGAGAC-3' }\end{array}$ & Johansen et al. (2005) \\
\hline hsp65 3' fragment & 1059 & $\begin{array}{l}\text { F: 5'-CGGTTCGACAAGGGTTACAT-3' } \\
\text { R: 5'-ACGGACTCAGAAGTCCATGC-3' }\end{array}$ & Turenne et al. (2006) \\
\hline DT-6 & 187 & $\begin{array}{l}\text { F: 5'-ATGGCCGGGAGACGATCTATGCCGGCGTAC-3' } \\
\text { R: 5'-CGTTCGATCGCAGTTTGTGCAGCGCGTACA-3' }\end{array}$ & Thierry et al. (1993) \\
\hline
\end{tabular}


Table 2. Distribution of $h s p 65$ sequevar types among M. avium isolates from different sources

hsp65 sequevar code 4 indicates M. avium subsp. avium/silvaticum; codes 1, 2, 3 and 15 indicate 'M. avium subsp. hominissuis' (Alvarez et al., 2008; Turenne et al., 2006); codes 16 and 17 indicate 'M. avium subsp. hominissuis' in this study.

\begin{tabular}{|c|c|c|c|c|c|c|c|c|c|}
\hline \multirow[t]{2}{*}{$h s p 65$ code } & \multicolumn{7}{|c|}{ Nucleotide at indicated base pair position (hsp65) } & \multicolumn{2}{|c|}{ Source } \\
\hline & 645 & 928 & 1128 & 1218 & 1269 & 1277 & 1536 & Sputum $(n)^{\star}$ & Other $(n) \dagger$ \\
\hline Code 2 & $\mathrm{C}$ & $\mathrm{C}$ & $\mathrm{C}$ & G & G & $\mathrm{C}$ & G & 25 & 2 \\
\hline Code 3 & $\mathrm{C}$ & $\mathrm{C}$ & G & G & $\mathrm{C}$ & G & G & 2 & 1 \\
\hline Code 4 & $\mathrm{~T}$ & $\mathrm{C}$ & G & G & $\mathrm{C}$ & G & G & 0 & 0 \\
\hline Code 17 & $\mathrm{C}$ & $\mathrm{C}$ & $\mathrm{C}$ & G & G & $\mathrm{C}$ & A & 4 & 0 \\
\hline
\end{tabular}

${ }^{\star}$ Sputum isolates from clinical patients with chronic obstructive pulmonary disease.

$\dagger$ Isolates from blood or lymph nodes.

with the restriction endonuclease PvuII and then subjected to electrophoresis on a $1 \%$ agarose gel (Takara), transferred to Hybond-N + (GE Healthcare), and detected with a DIG Luminescent Detection kit (Roche). DIG-labelled marker II (Roche) was used as an internal DNA size marker. Finally, the membrane was exposed to X-ray film (GE Healthcare), and RFLP analysis was performed with Fingerprinting II (Bio-Rad Laboratories). After stripping of the DIG probe, we used the same membrane for probing for the DT-6 gene, which was reported (Thierry et al., 1993) as a M. avium-specific gene, to verify the performance of the assay.

Statistical analysis. Categorical data for the detection rate of insertion sequences were analysed statistically by the chi-square test.

\section{RESULTS AND DISCUSSION}

\section{Identification of clinical isolates}

All of the 114 clinical strains were identified as M. avium by using the COBAS AMPLICOR Mycobacterium Test. As a result of nucleotide sequence analysis of the $3^{\prime}$ fragment of the $h s p 65$ gene, all strains were identified as ' $M$. avium subsp. hominissuis' (Table 2), and two new sequevars of code 16 (GenBank accession no. AB453830) and code 17 (GenBank accession no. AB453831) were found. These codes, 16 and 17, differed from those of bird-type $M$. avium subsp. avium, because base no. 645 was substituted to $\mathrm{C}$ from $\mathrm{T}$ which was reported to be specific to $M$. avium subsp. avium.

\section{Distribution of insertion sequences}

PCR analysis for ' $M$. avium subsp. hominissuis'-specific insertion sequences showed that a relatively low detection rate of IS 1245 was one of the characteristics of clinical isolates in Japan, as reported previously (Kuwabara et al., 2004; Moriyama et al., 2006), and one IS1311-negative strain was newly identified (Table 3). Confirmation by Southern hybridization and reblotting with the same membrane for DT-6 showed that this isolate was truly IS1311-negative (Fig. 1a, b).

Three sputum isolates and one blood isolate that were negative for IS 1245 had hsp65 sequevar code 2 . It has been suggested that isolates lacking IS 1245 with $h s p 65$ sequevar

Table 3. Comparison of detection rates of insertion sequences in $M$. avium isolates recovered from sputum with those from other sites

Data are presented as $n(\%)$ or odds ratios (95\% confidence interval).

\begin{tabular}{|lcccc|}
\hline & Sputum $^{*}(\boldsymbol{n}=\mathbf{8 6})$ & Others $\dagger(\boldsymbol{n}=\mathbf{2 8})$ & OR $(\mathbf{9 5} \% \mathbf{C I}) \neq$ & $\boldsymbol{P}$-value \\
\hline IS1245 & $70(81.3)$ & $26(92.9)$ & $0.34(0.07-1.57)$ & 0.15 \\
IS1311 & $85(98.8)$ & $28(100)$ & 1.00 & 0.57 \\
IS901/ISMav6\$ & $64(74.4)$ & $12(42.9)$ & $3.88(1.59-9.46)$ & 0.002 \\
\hline
\end{tabular}

${ }^{\star}$ Sputum isolates from clinical patients with chronic obstructive pulmonary disease.

$\dagger$ Isolates from blood or lymph nodes.

‡OR, Odds ratios; CI, confidence interval.

§IS901 is likely to be uncommon and the high level detected with the IS901 probe is likely to represent primarily ISMav6. 
(a)

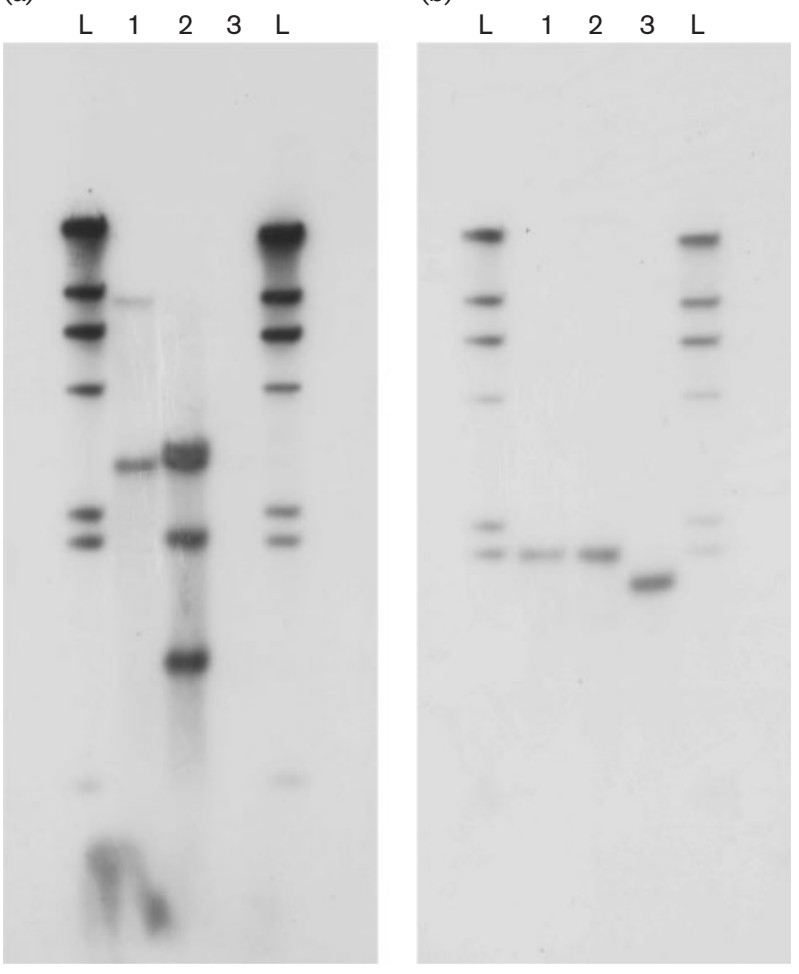

Fig. 1. Southern blot analysis for detection of IS1311. Total DNA was digested with restriction endonuclease Pvull and blotted with an IS1311-specific probe (a) and DT-6 probe as a positive control (b). Lanes: L, molecular mass ladder; 1, GTC00603 (M. avium subsp. avium); 2, ATCC 19978 ('M. avium subsp. hominissuis'); 3, lung-av-002 (IS1311-negative strain). code 2 might represent environmental contamination (Alvarez et al., 2008). However, taking into account the fact that all the patients from whom such strains were detected in the present study had symptoms of infection, and that specimens were handled carefully according to laboratory quality control standards, contamination was unlikely. Moreover, no strains with the same genotype were identified during the same period when $M$. avium isolates lacking IS 1245 were detected.

In the clinical environment, we found that the $h s p 65$ gene analysis was useful to identify the subspecies of M. avium, especially when there were substantial numbers of isolates with no subspecies-specific insertion sequences.

PCR analysis for IS901 showed a unique distribution among Japanese M. avium isolates (Table 3). Although Pavlik et al. (2000) reported that strains possessing IS901 were isolated from $12(8 \%)$ out of 152 human isolates, IS 901 has either been detected at a very low frequency in human isolates or not detected at all (Alvarez et al., 2008; Möbius et al., 2006; Ritacco et al., 1998). Therefore the detection rate of IS901 in Japan was significantly higher than in other countries.

\section{Characterization of a newly detected insertion sequence}

The high frequency of detection of IS 901 by PCR in $M$. avium clinical isolates prompted us to conduct nucleotide sequence analysis on this element. Compared with the nucleotide sequence of the original bird-type IS901, 60 point mutations were found in the sequence of the insertion sequence detected from the clinical isolates (Supplementary Fig. S1 in JMM Online). These mutations were identical in all PCR-positive clinical isolates. The

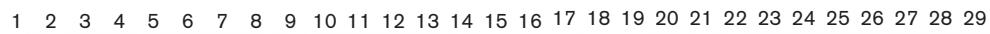

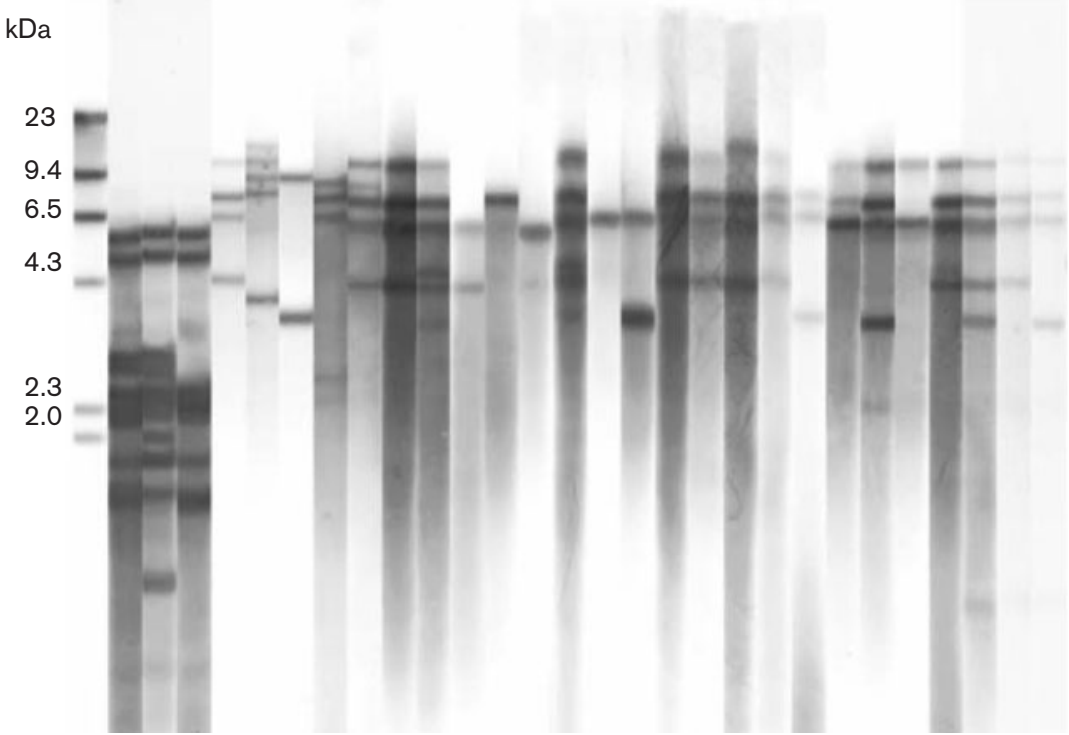

Fig. 2. RFLP analysis of IS901 after digestion with restriction endonuclease Pvull. Lanes: 1, molecular mass ladder; 2, GTC00603; 3, ATCC 49884; 4, ATCC 35718; 5-29, clinical isolates. Lanes 2-4 were detected by the IS 901 probe and lanes $5-29$ by the ISMav6 probe. 
sequence identity with the original IS 901 was $95 \%$. Therefore, this new insertion sequence was designated ISMav6 (GenBank accession no. AB447556). The presence of ISMav6 in the sequenced M. avium 104 strain was examined by PCR with three sets of primers (primers IS901-1F and IS901-1R, IS901-2F and IS901-2R, and IS9013F and IS901-3R) (Table 1). No PCR amplifications for ISMav6 were found with these primers sets, which was consistent with the fact that there is no gene corresponding to ISMav6 in the genome database of M. avium 104 (accession no. CP000479, data not shown). ISMav6 encoded a putative protein of 403 amino acids, which showed $96 \%$ identity with that encoded by IS 901 . The PCR for FR300, which was supposed to amplify IS901 together with the flanking regions of the insertion sites, gave a $1776 \mathrm{bp}$ band for bird-type standard strains containing IS901. However, a $300 \mathrm{bp}$ band was detected for M. avium 104, ATCC 19978 and all clinical strains, indicating no insertion sequence at the insertion sites for IS901 (data not shown).

The hybridization profile with the probe derived from IS901 appeared to be quite similar to that with the probe prepared from ISMav6 of the clinical isolates, indicating cross-reactivity of the IS901-type probe with ISMav6 in clinical isolates. RFLP analysis with the IS901 probe revealed that its copy number in the bird-type strains was 10-13, while that of ISMav6 in the clinical isolates was 0-6 (Fig. 2), showing relatively infrequent insertions at sites away from FR300. This new insertion sequence, possibly specific to ' $M$. avium subsp. hominissuis', was detected at a significantly higher frequency in the clinical isolates recovered from sputum than in those recovered from blood or lymph nodes (Table 3).

As IS901 is reported to have an influence on the pathogenicity of $M$. avium subsp. avium (Kunze et al., 1991; Pavlik et al., 2000), ISMav6 may also be related to the pathogenicity of ' $M$. avium subsp. hominissuis', especially in strains causing pulmonary disease.

In conclusion, clinical $M$. avium infections in Japan are caused by ' $M$. avium subsp. hominissuis', as is the case in other countries. Sixteen per cent of the Japanese 'M. avium subsp. hominissuis' isolates lacked IS1245 and one isolate lacked IS1311. A new insertion sequence, ISMav6, which is homologous to the original bird-type IS901, was found frequently in the Japanese clinical isolates, especially those recovered from sputum. The presence of ISMav6 may be one of the bacterial factors that enhances pathogenicity and contributes to the increase of $M$. avium pulmonary infections in Japan.

\section{ACKNOWLEDGEMENTS}

Thanks are due to $\mathrm{Mr}$ Takami Shibayama (National Hospital Organization, Higashinagoya National Hospital) for technical support, to Dr Teruo Kirikae (International Medical Center of Japan) for generously providing the clinical strains, and to Professor William Bishai, Ms Deborah Geiman (Johns Hopkins School of Medicine) and
Dr Takemasa Takii (Nagoya City University) for generously providing the M. avium 104 strain.

\section{REFERENCES}

Alvarez, J., García, I. G., Aranaz, A., Bezos, J., Romero, B., de Juan, L., Mateos, A., Gómez-Mampaso, E. \& Domínguez, L. (2008). Genetic diversity of Mycobacterium avium isolates recovered from clinical samples and from the environment: molecular characterization for diagnostic purposes. J Clin Microbiol 46, 1246-1251.

Chacon, O., Bermudez, L. E. \& Barletta, R. G. (2004). Johne's disease, inflammatory bowel disease, and Mycobacterium paratuberculosis. Annu Rev Microbiol 58, 329-363.

Dvorska, L., Bull, T. J., Bartos, M., Matlova, L., Svastova, P., Weston, R. T., Kintr, J., Parmova, I., van Soolingen, D. \& Pavlik, I. (2003). A standardized restriction fragment length polymorphism (RFLP) method for typing Mycobacterium avium isolates links IS901 with virulence for birds. J Microbiol Methods 55, 11-27.

Harris, N. B. \& Barletta, R. G. (2001). Mycobacterium avium subsp. paratuberculosis in veterinary medicine. Clin Microbiol Rev 14, 489512.

Henry, M. T., Inamdar, L., O'Riordain, D., Schweiger, M. \& Watson, J. P. (2004). Nontuberculous mycobacteria in non-HIV patients: epidemiology, treatment and response. Eur Respir J 23, 741-746.

Johansen, T. B., Djonne, B., Jensen, M. R. \& Olsen, I. (2005). Distribution of IS1311 and IS 1245 in Mycobacterium avium subspecies revisited. J Clin Microbiol 43, 2500-2502.

Kunze, Z. M., Wall, S., Appelberg, R., Silva, M. T., Portaels, F. \& Mcfadden, J. J. (1991). IS901, a new member of a widespread class of atypical insertion sequences, is associated with pathogenicity in Mycobacterium avium. Mol Microbiol 5, 2265-2272.

Kunze, Z. M., Portaels, F. \& Mcfadden, J. J. (1992). Biologically distinct subtypes of Mycobacterium avium differ in possession of insertion sequence IS901. J Clin Microbiol 30, 2366-2372.

Kuwabara, K., Watanabe, Y., Wada, K. \& Tsuchiya, T. (2004). Relations between clinical subtypes of Mycobacterium avium pulmonary disease and polyclonal infections detected by IS1245 based restriction fragment length polymorphism analysis. Kekkaku 79, 3946 (in Japanese).

Marras, T. K. \& Daley, C. L. (2002). Epidemiology of human pulmonary infection with nontuberculous mycobacteria. Clin Chest Med 23, 553-567.

Mijs, W., de Haas, P., Rossau, R., Van der Laan, T., Rigouts, L., Portaels, F. \& van Soolingen, D. (2002). Molecular evidence to support a proposal to reserve the designation Mycobacterium avium subsp. avium for bird-type isolates and ' $M$. avium subsp. hominissuis' for the human/porcine type of M. avium. Int J Syst Evol Microbiol 52, 1505-1518.

Möbius, P., Lentzsch, P., Moser, I., Naumann, L., Martin, G. \& Köhler, H. (2006). Comparative macrorestriction and RFLP analysis of Mycobacterium avium subsp. avium and Mycobacterium avium subsp. hominissuis isolates from man, pig, and cattle. Vet Microbiol 117, 284291.

Moriyama, M., Ogawa, K., Nishimori, K., Uchiya, K., Ito, T., Yagi, T., Nakashima, I., Nakagawa, T., Tarumi, O. \& Nikai, T. (2006). Usefulness of variable numbers of tandem repeats typing in clinical strains of Mycobacterium avium. Kekkaku 81, 559-566 (in Japanese).

Nishimori, K., Eguchi, M., Nakaoka, Y., Onodera, Y., Ito, T. \& Tanaka, K. (1995). Distribution of IS901 in strains of Mycobacterium avium complex from swine by using IS901-detecting 
primers that discriminate between $M$. avium and Mycobacterium intracellulare. J Clin Microbiol 33, 2102-2106.

Pavlik, I., Svastova, P., Bartl, J., Dvorska, L. \& Rychlik, I. (2000). Relationship between IS901 in the Mycobacterium avium complex strains isolated from birds, animals, humans, and the environment and virulence for poultry. Clin Diagn Lab Immunol 7, 212-217.

Ritacco, V., Kremer, K., Van der Laan, T., Pijnenburg, J. E., de Haas, P. E. \& van Soolingen, D. (1998). Use of IS 901 and IS1245 in RFLP typing of Mycobacterium avium complex: relatedness among serovar reference strains, human and animal isolates. Int J Tuberc Lung Dis 2, 242-251.
Sakatani, M. (1999). Nontuberculous mycobacteriosis: the present status of epidemiology and clinical studies. Kekkaku 74, 377-384 (in Japanese).

Thierry, D., Vincent, V., Clément, F. \& Guesdon, J. L. (1993). Isolation of specific DNA fragments of Mycobacterium avium and their possible use in diagnosis. J Clin Microbiol 31, 1048-1054.

Turenne, C. Y., Semret, M., Cousins, D. V., Collins, D. M. \& Behr, M. A. (2006). Sequencing of $h s p 65$ distinguishes among subsets of the Mycobacterium avium complex. J Clin Microbiol 44, 433-440.

Turenne, C. Y., Wallace, R., Jr \& Behr, M. A. (2007). Mycobacterium avium in the postgenomic era. Clin Microbiol Rev 20, 205-229. 